\title{
Floquet engineering of multifold fermions
}

\author{
Rimika Jaiswal 10 \\ Undergraduate Programme, Indian Institute of Science, Bangalore 560012, India \\ Awadhesh Narayan (1)* \\ Solid State and Structural Chemistry Unit, Indian Institute of Science, Bangalore 560012, India
}

(Received 7 September 2020; accepted 1 December 2020; published 15 December 2020)

\begin{abstract}
Using Floquet theory, we investigate the effect of light on triplefold fermions. We study a low-energy model as well as a simplified tight-binding model under illumination. We find that the threefold degeneracy remains symmetry protected even after applying light if the original band structure is rotationally symmetric around the degeneracy. Otherwise, light can lift the degeneracy and open up a gap. We further investigate the effect of light on the topological Fermi arcs by means of numerical computations. The changes caused by illumination are reflected in experimentally detectable signatures, such as the anomalous Hall conductivity, which we calculate.
\end{abstract}

DOI: 10.1103/PhysRevB.102.245416

\section{INTRODUCTION}

In recent decades, topological aspects of physics have been in the limelight. Starting with the discovery of the quantum Hall effect [1,2], ideas from topology have started to permeate condensed-matter and materials physics. More recently, the proposal and subsequent discovery of topological insulators and superconductors has led to intense activity [3,4]. Building on these ideas, the concept of topological semimetals was proposed. They are a class of materials which harbor topologically stable energy bands crossing near the Fermi surface. It was found that in materials such as graphene, the low-energy behavior around such degeneracies resembles the relativistic Dirac equation. Soon, condensed-matter systems became a basis for the discovery and realization of fermionic particles predicted in high-energy physics such as Majorana fermions and Dirac and Weyl fermions [5,6].

Free fermionic excitations in topological semimetals with no high-energy counterparts were recently discovered [7]. These materials, commonly called multifold semimetals, are characterized by higher order (larger than 2) band crossings at the degenerate points. Experiments have confirmed the presence of such higher order degeneracies in materials such as $\mathrm{RhSi}$ [8], CoSi [9,10], AlPt [11], and the superconducting metal $\mathrm{PdSb}_{2}$ [12]. These classes of materials exhibit several remarkable properties. Multifold fermions have an enhanced linear response (compared to Weyl fermions) due to their higher topological charge [13]. They also show a unique quantized photogalvanic effect [14], which has been very recently detected in experiments [15-17].

Floquet engineering [18], or the controlling of topological transitions using periodic drives such as light, has recently become an interesting field of study $[19,20]$. Starting from the work of Oka and Aoki, who discovered that applying light

*awadhesh@iisc.ac.in on graphene makes it behave like a topological insulator [21], various theoretical studies [22-26] and experiments [27-29] have illustrated how topological phases can be generated by the periodic driving of systems with light. Floquet engineering provides a way of experimentally realizing topological phases, with excellent tunability. Promising ways of experimentally realizing tunable Weyl semimetals by shining light on Dirac semimetals [30], topological insulators [31], or nodal line semimetals [32-34] have been demonstrated. More recently, Floquet theory has also been used to propose possible realizations of fractional Chern insulators [35] and topological superconductors [36]. Possible applications of Floquet theory to generate topological phases in bilayer graphene [37], photonic systems [38,39], and non-Hermitian systems [40] are also being explored.

Motivated by these developments, here we study the effect of light on triplefold fermions using the Floquet formalism, based on an effective low-energy model, as well as a simplified tight-binding model. We show that elliptically polarized light can cause a band gap to open up and/or shift the threefold degeneracy depending on the intrinsic symmetries of the system. We map out the complete phase diagram of triplefold fermions under illumination and calculate the resulting anomalous Hall signatures. We also investigate the effect of light on topological Fermi arcs by means of numerical computations employing a simplified tight-binding model. Our predicted signatures can be experimentally detected and provide insights into the interaction between light and multifold fermions. We hope to motivate further exploration of the topological phases that can arise from the periodic driving of multifold semimetals.

\section{EFFECTIVE LOW-ENERGY MODEL}

We consider the general low-energy Hamiltonian around a threefold degenerate point as derived by Bradlyn et al. [7]. Taking the degenerate point, $k_{0}$, to be at the origin, the 


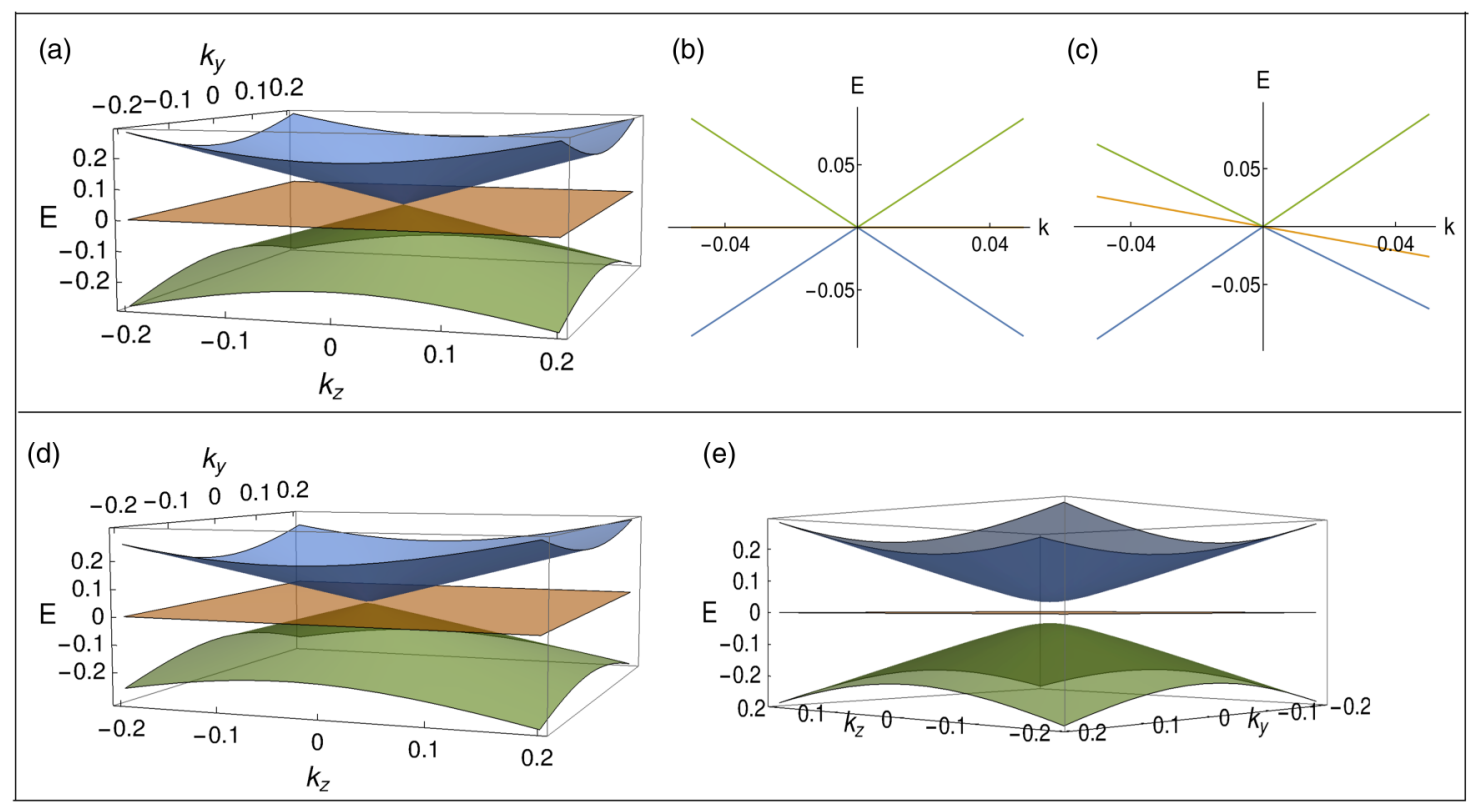

FIG. 1. Band-structure plots illustrating the effect of light on threefold fermions. Top: Band structure before illumination. Bottom: Band structure after illumination. For panels (a), (d), and (e), the eigenvalues are plotted as a function of $k_{y}$ and $k_{z}$ (at $k_{x}=0$ ). Panel (a) displays the threefold degeneracy at $\mathbf{k}=0$. Panels (b) and (c) show the dispersion along $k_{x}=k_{y}=k_{z}$ for (b) $\phi=\pi / 6$ and (c) $\phi=\pi / 12$. The value of $\phi$ determines whether the threefold crossing is centrosymmetric (rotationally symmetric about the degeneracy) or not. Panels (d) and (e) show the resultant band structure after illumination for $\phi=\pi / 2$ and $\phi=0$, respectively. For $\phi=\pi / 2$, the degeneracy remains intact but shifts along $k_{z}$ whereas for $\phi=0$ a band gap opens up. Here, we choose the following parameter values: $A_{x}=2, A_{y}=1, \omega=30$, and $\theta=\pi / 2$. In our chosen units, $k$ is dimensionless and $E$ is expressed in units of $\hbar v_{f}$.

Hamiltonian reads

$$
H_{3 f}(\mathbf{k})=E_{0}+\hbar v_{f}\left[\begin{array}{ccc}
0 & e^{i \phi} k_{x} & e^{-i \phi} k_{y} \\
e^{-i \phi} k_{x} & 0 & e^{i \phi} k_{z} \\
e^{i \phi} k_{y} & e^{-i \phi} k_{z} & 0
\end{array}\right],
$$

where $E_{0}$ is an energy offset, $\hbar$ is the reduced Planck's constant, $v_{f}$ is the effective velocity around $\mathbf{k}=0$, and $\phi$ is a material-dependent parameter. We shall consider the threeband touching point (3-BTP) to be at the Fermi energy and thus set $E_{0}=0$. From here on, we also work in units where $\hbar, v_{f}$, and $e$ are unity for simplicity.

One can observe that for $\phi=\pi / 6(\bmod \pi / 3)$, i.e., for $\phi=\pi / 6, \pi / 2, \ldots$, the Hamiltonian takes the form $H=\mathbf{k} \cdot \boldsymbol{S}$, where $S$ is the spin-1 representation of $\mathrm{SU}(2)$ as given in Eq. (24). The corresponding eigenvalues read $E=0, \pm k$, showing that the band structure is completely centrosymmetric, i.e., having complete rotational symmetry about the degeneracy at $\mathbf{k}=0$. The band structures illustrating this are shown in Fig. 1.

Away from these $\phi$ values, the conical bands tilt and the centrosymmetry about the degeneracy vanishes. The tilt is maximum for $\phi=0(\bmod \pi / 3)$, i.e., for $\phi=0, \pi / 3$, $2 \pi / 3, \ldots$.

The upper and lower bands are topologically nontrivial, having Chern numbers \pm 2 . The flat band in the middle is, however, trivial with Chern number zero. At half filling, the 3-BTP acts as a source of Berry curvature with charge +2 for $\phi \in\left(0, \frac{\pi}{3}\right)$, whereas it acts as a sink of Berry curvature with charge -2 for $\phi \in\left(\frac{\pi}{3}, \frac{2 \pi}{3}\right)$ and this pattern continues periodically. However, for $\phi=n \frac{\pi}{3}, n \in \mathbb{Z}$, the eigenvalue spectrum is degenerate along lines corresponding to $\left|k_{x}\right|=\left|k_{y}\right|=\left|k_{z}\right|$ and the Chern number is not defined. These $\phi$ points with extended degeneracy can be thought to partition the space of Hamiltonians into distinct phases that differ in their Fermi surface topology.

\section{A. Floquet analysis for the low-energy model}

We next consider shining light on a material with threefold crossing. If a system is subjected to an external timedependent periodic perturbation such that $H(t+T)=H(t)$, then the Floquet approximation states that in the high frequency $(\omega)$ limit, an effective Hamiltonian describing the system can be written as $[18,20]$

$$
H_{\text {floq }}(\mathbf{k})=H_{0}(\mathbf{k})+\frac{\left[H_{+1}(\mathbf{k}), H_{-1}(\mathbf{k})\right]}{\omega}+\mathcal{O}\left(\frac{1}{\omega^{2}}\right),
$$

where $\omega=2 \pi / T$ and $H_{m}(\mathbf{k})=\frac{1}{T} \int_{0}^{T} H(\mathbf{k}, t) e^{-i m \omega t} d t$. In cases where the Hamiltonian $H(t)$ can be separated into a time-independent and a time-dependent part such that $H(\mathbf{k}, t)=H_{0}(\mathbf{k})+V(\mathbf{k}, t)$, the expression simplifies to

$$
H_{\text {floq }}(\mathbf{k})=H_{0}(\mathbf{k})+\frac{\left[V_{+1}(\mathbf{k}), V_{-1}(\mathbf{k})\right]}{\omega}+\mathcal{O}\left((V / \omega)^{2}\right) .
$$

If light is applied on our threefold fermion system, we can use Floquet theory to study the resulting effective band structure if the frequency of light, $\omega$, is sufficiently large such that $\omega \gg V$ (see Appendix A). We denote the vector potential of applied light by $\boldsymbol{A}(t)$. For noncavity modes, light is plane-polarized, allowing us to choose $A_{z}=0$. Thus with a phase difference $\theta$ between the $x$ and $y$ components, we have 
$\boldsymbol{A}=\left[A_{x} \cos (\omega t), A_{y} \cos (\omega t+\theta), 0\right]$. The effect of this vector potential on our Hamiltonian can be incorporated through the standard Peierls substitution, i.e., $\mathbf{k} \rightarrow \mathbf{k}+\boldsymbol{A}$. This gives $H_{3 f}(\mathbf{k}, t)=H_{3 f}(\mathbf{k})+V(t)$, where

$$
V(t)=\left[\begin{array}{ccc}
0 & e^{i \phi} A_{x} \cos \omega t & e^{-i \phi} A_{y} \cos (\omega t+\theta) \\
e^{-i \phi} A_{x} \cos \omega t & 0 & 0 \\
e^{i \phi} A_{y} \cos (\omega t+\theta) & 0 & 0
\end{array}\right] .
$$

Now, for large $\omega$, we can use the Floquet approximation given by Eq. (3) to study the effective photon-dressed static Hamiltonian $H_{\text {floq }}$. After integrating over one time period, we obtain the form of the Fourier components as

$$
\begin{aligned}
V_{+1}(t) & =\frac{1}{2}\left[\begin{array}{ccc}
0 & e^{i \phi} A_{x} & e^{-i \phi} e^{i \theta} A_{y} \\
e^{-i \phi} A_{x} & 0 & 0 \\
e^{i \phi} e^{i \theta} A_{y} & 0 & 0
\end{array}\right], \\
V_{-1}(t) & =\frac{1}{2}\left[\begin{array}{ccc}
0 & e^{i \phi} A_{x} & e^{-i \phi} e^{-i \theta} A_{y} \\
e^{-i \phi} A_{x} & 0 & 0 \\
e^{i \phi} e^{-i \theta} A_{y} & 0 & 0
\end{array}\right] .
\end{aligned}
$$

This gives the light-induced term as

$$
\begin{aligned}
\frac{\left[V_{+1}, V_{-1}\right]}{\hbar \omega} & =\frac{i A_{x} A_{y} \sin \theta}{2 \omega}\left[\begin{array}{ccc}
0 & 0 & 0 \\
0 & 0 & -e^{-i 2 \phi} \\
0 & e^{+i 2 \phi} & 0
\end{array}\right] \\
& =i \gamma\left[\begin{array}{ccc}
0 & 0 & 0 \\
0 & 0 & -e^{-i 2 \phi} \\
0 & e^{+i 2 \phi} & 0
\end{array}\right],
\end{aligned}
$$

where we have defined the prefactor $\gamma$, having the same dimensions as $k$, as

$$
\gamma=\frac{A_{x} A_{y} \sin \theta}{2 \omega} .
$$

Note that because of the $\sin \theta$ factor, $\gamma$ and hence the light-induced term vanishes for linearly polarized light (i.e., for $\theta=0$ ), while taking the maximum value for $\theta=\pi / 2$ (elliptically polarized light). We can understand this as follows. Elliptical polarization provides the chirality needed to break time-reversal symmetry. In contrast, a linear polarization, made of equal superposition of clockwise and anticlockwise circular polarizations, cannot break time-reversal symmetry and hence does not lead to observable changes.

\section{B. Changes in the band structure}

Our effective Floquet Hamiltonian after applying light thus reads

$$
H_{\text {floq }}=\left[\begin{array}{ccc}
0 & e^{i \phi} k_{x} & e^{-i \phi} k_{y} \\
e^{-i \phi} k_{x} & 0 & e^{i \phi} k_{z}-i \gamma e^{-2 i \phi} \\
e^{i \phi} k_{y} & e^{-i \phi} k_{z}+i \gamma e^{2 i \phi} & 0
\end{array}\right] .
$$

For certain cases, this Hamiltonian can be mapped back to the original low-energy model, $H_{3 f}$ for threefold fermions. For $\phi=\frac{\pi}{6}(\bmod \pi / 3)$, we can write $H_{\text {floq }}(\mathbf{k})=H_{3 f}(\tilde{\mathbf{k}})$, where $\tilde{\mathbf{k}}=\left(k_{x}, k_{y}, k_{z}-\gamma \sin 3 \phi\right)$. The system in the presence of light is thus exactly like the unperturbed system but with the
3-BTP now shifted along the $k_{z}$ axis. We now find the new eigenvalues,

$$
E=0, \pm \sqrt{k_{x}^{2}+k_{y}^{2}+\left(k_{z}-\gamma \sin 3 \phi\right)^{2}},
$$

which are degenerate at $\mathbf{k}=(0,0, \gamma \sin 3 \phi)$. In contrast, however, for $\phi=0(\bmod \pi / 3)$, we observe that a band gap opens up at $\mathbf{k}=0$ (see Fig. 1). For other intermediate values of $\phi$, we find that a band gap opens up along with a shift in the 3-BTP. For $\phi \in\left(0, \frac{\pi}{3}\right)$, it shifts to the right, while for $\phi \in\left(\frac{\pi}{3}, \frac{2 \pi}{3}\right)$ it shifts to the left, and this alternating pattern continues in a periodic manner.

Next, we find the analytical expressions for the lightinduced band gap $\left(\Delta_{g}\right)$ and location of the 3-BTP after the shift $\left(k_{z}^{0}\right)$. The eigenvalues of $H_{\text {floq }}$ satisfy the following equation:

$$
E^{3}-E\left(\gamma^{2}+k^{2}-2 \gamma k_{z} \sin 3 \phi\right)-2 k_{x} k_{y} k_{z} \cos 3 \phi=0 .
$$

The exact form of the eigenvalues for any general $\phi$ can be derived (given in Appendix B), using which we obtain

$$
\Delta_{g}=2 \gamma \cos 3 \phi \quad \text { and } \quad k_{z}^{0}=\gamma \sin 3 \phi .
$$

These expressions succinctly summarize the trends described earlier (see Fig. 2). For $\phi=0(\bmod \pi / 3)$ we obtain the largest band-gap opening $\Delta_{g}=2 \gamma$ at $\mathbf{k}=0$, while for $\phi=\pi / 6(\bmod \pi / 3)$, we find the maximum shift of the 3-BTP to $k_{z}=\gamma$. The $\cos 3 \phi, \sin 3 \phi$ nature of variation of the band

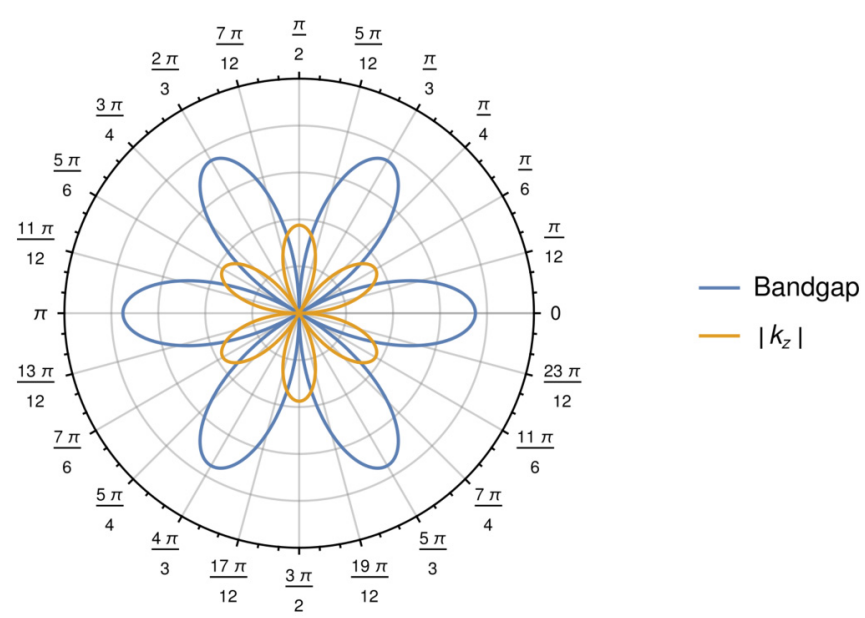

FIG. 2. Angular plot depicting the variation of the band gap $\left(\Delta_{g}\right)$ and the shift of the threefold degeneracy $\left(\left|k_{z}\right|\right)$ with $\phi$. There is a $\pi / 3$ periodicity in the response of the system. For $\phi=0(\bmod \pi / 3)$, a maximum band gap opens up with the shift being zero. Whereas, for $\phi=\pi / 6(\bmod \pi / 3)$, there is a maximum shift along $k_{z}$ but with no band-gap opening. Here we set $\gamma=1 / 30$. In our chosen units, $k_{z}$ is dimensionless and $\Delta_{g}$ is expressed in units of $\hbar v_{f}$. 
gap and shift, respectively, also captures the inherent $\pi / 3$ periodicity in the response of the system. We note that the results recently reported in the literature [41] for the special case of $\phi=\pi / 2$ are consistent with ours.

Interestingly, we observe the maximum shift of the 3BTP along the $k_{z}$ axis, with the threefold degeneracy being maintained, for cases where the original band structure was fully centrosymmetric around the degeneracy. Analogously, we observe the maximum band gap opening for cases where the original band structure showed the maximal tilt along the $(1,1,1)$ direction. This matches Weyl semimetal studies where it has been shown that lifting the degeneracy in a fully centrosymmetric Weyl semimetal is difficult [42]. It has also been shown that tilted Weyl semimetals have a better response to light, and they can support significant photocurrents while centrosymmetric Weyls cannot [43].

\section{Effect of higher order terms}

For our low-energy model under illumination, all multiphoton terms $H_{m}$ for $m \neq 0,1,-1$ are zero. The only nonzero terms in the Floquet-Magnus expansion thus have the form $\left[H_{0}, H_{ \pm 1}\right] / \omega,\left[H_{ \pm 1},\left[H_{0}, H_{\mp 1}\right]\right] / \omega^{2}$ and so on. All the properties discussed above hold true even when these higher-order terms are considered.

\section{Symmetry analysis}

We discovered that depending on the value of $\phi$, the threefold degeneracy can either be lifted or shifted upon illumination with elliptically polarized light. To understand the underlying reason, it is insightful to look at the symmetries of our system and see if they change after applying light. Multifold degeneracies in space group 199 are characterized by three unitary symmetries which protect the threefold degeneracy: $\left\{C_{2 x} \mid \frac{1}{2} \frac{1}{2} 0\right\},\left\{C_{2 y} \mid 0 \frac{1}{2} \frac{\overline{1}}{2}\right\}$ and $\left\{C_{3,111}^{-1} \mid 101\right\}$. They can be chosen to be [7]

$$
\begin{aligned}
G_{1} & =\left[\begin{array}{rrr}
-1 & 0 & 0 \\
0 & -1 & 0 \\
0 & 0 & 1
\end{array}\right], G_{2}=\left[\begin{array}{rrr}
0 & 0 & -1 \\
1 & 0 & 0 \\
0 & -1 & 0
\end{array}\right], \\
G_{3} & =\left[\begin{array}{lll}
0 & 0 & 1 \\
1 & 0 & 0 \\
0 & 1 & 0
\end{array}\right] .
\end{aligned}
$$

A Hamiltonian obeying these symmetries is constrained to satisfy the following conditions:

$$
G_{i} H(k) G_{i}^{-1}=H\left[D\left(G_{i}\right) k\right], \quad i=1,2,3,
$$

where the matrices $D\left(G_{i}\right)$ have the representation

$$
\begin{aligned}
& D\left(G_{1}\right)=\left[\begin{array}{rrr}
1 & 0 & 0 \\
0 & -1 & 0 \\
0 & 0 & -1
\end{array}\right], \quad D\left(G_{2}\right)=\left[\begin{array}{rrr}
0 & -1 & 0 \\
0 & 0 & 1 \\
-1 & 0 & 0
\end{array}\right], \\
& D\left(G_{3}\right)=\left[\begin{array}{lll}
0 & 1 & 0 \\
0 & 0 & 1 \\
1 & 0 & 0
\end{array}\right] .
\end{aligned}
$$

It is easy to verify that our original Hamiltonian $H_{3 f}$ satisfies Eq. (13). Next, we investigate whether these symmetries are preserved once light is applied. We find that Eq. (13) is satisfied by $H_{\text {floq }}(\tilde{\mathbf{k}})$ with $\tilde{\mathbf{k}}=\left(k_{x}, k_{y}, k_{z}-\gamma \sin 3 \phi\right)$, whenever $\phi=\frac{\pi}{6}(\bmod \pi / 3)$. This implies that for these values of $\phi$, the threefold degeneracy shifts but remains symmetry protected even after illumination. On the other hand, for all other values of $\phi, H_{\text {floq }}(\mathbf{k})$ fails to satisfy the symmetry conditions. The degeneracy is no longer symmetry protected and can be lifted by light, leading to opening of a band gap. Note that because both circular and elliptically polarized light break all three lattice symmetries, the results are similar for both these choices of polarization. Thus, we can understand our results based on these symmetry arguments.

\section{Illustration of band structure engineering with densities of states}

Having discussed the tuning of the band structure with light, we next illustrate these changes using the k-resolved density of states (DOS) which can be directly measured in angle-resolved photoemission (ARPES) experiments. The DOS at an energy $E$ given by $\rho(E, \mathbf{k})$ can be calculated using [44]

$$
\rho(E, \mathbf{k})=-\frac{1}{\pi} \Im(\operatorname{Tr}(G(E, \mathbf{k}))),
$$

where the energy-dependent Green's function $G(E, \mathbf{k})$ is given by

$$
G(E, \mathbf{k})=\left(E+i \eta-H_{\text {floq }}(\mathbf{k})\right)^{-1}
$$

where $\eta \rightarrow 0^{+}$is a positive infinitesimal. Contour plots for $\rho(E, \mathbf{k})$ obtained are presented in Fig. 3. For the unperturbed system, we observe that the DOS for $E$ close to the Fermi energy is large near $\mathbf{k}=0$ due to the degeneracy but decreases gradually as we move away from it. (Note that exactly at $E=$ 0 , the DOS would be very large due to the contributions from the flatband.) When light is applied, the high density point representing the threefold degeneracy shifts along $k_{z}$ for $\phi=$ $\pi / 6(\bmod \pi / 3)$ while, for other $\phi$ values, the high DOS near the center vanishes as the degeneracy is no longer present and a band gap opens up. The dependence of $\Delta_{g}$ and $k_{z}^{0}$ on the intensity of applied light can also be clearly seen from our results.

\section{E. Anomalous Hall conductivity}

The signatures of light-induced changes to threefold fermions could be observed in several experimental probes. We propose measurement of the anomalous Hall effect as a direct way to verify the light-induced changes. As we discussed before, for $\phi=\pi / 6(\bmod \pi / 3)$, we can write $H_{\text {floq }}(\mathbf{k})=$ $H_{3 f}(\mathbf{k}-\delta \mathbf{k})$ where $\delta \mathbf{k}=(0,0, \gamma \sin 3 \phi)$. A band gap does not open up at these values of $\phi$ and the 3-BTP continues to behave like a source or sink of Berry curvature with monopole charge \pm 2 . Moreover, since the flatband is topologically trivial with Chern number zero, for these particular values of $\phi$, we can calculate the change in the anomalous Hall 
(a)

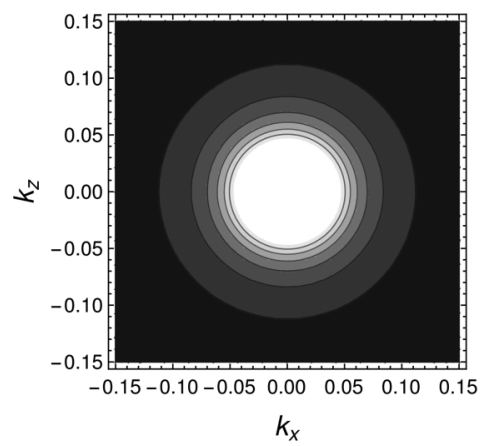

(d)

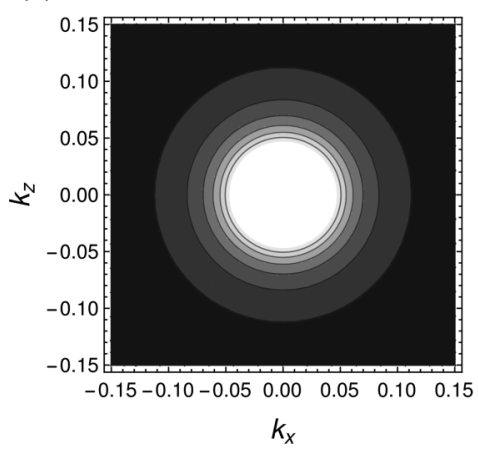

(b)

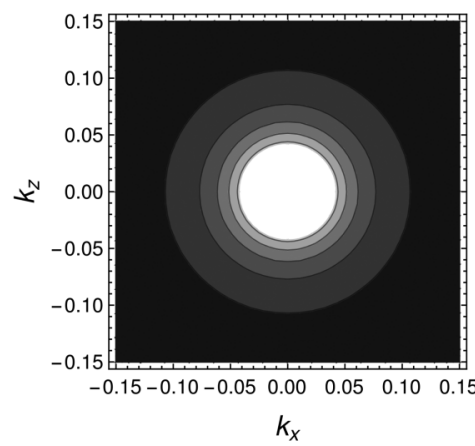

(e)

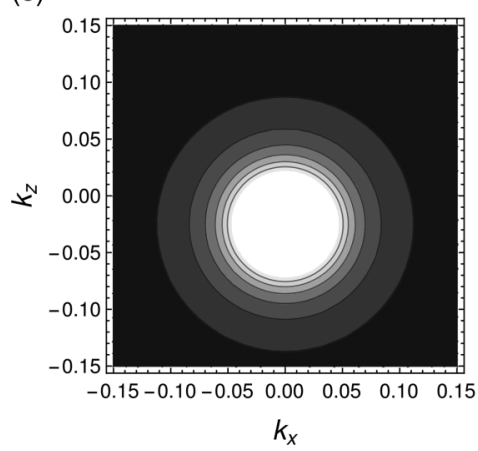

(c)

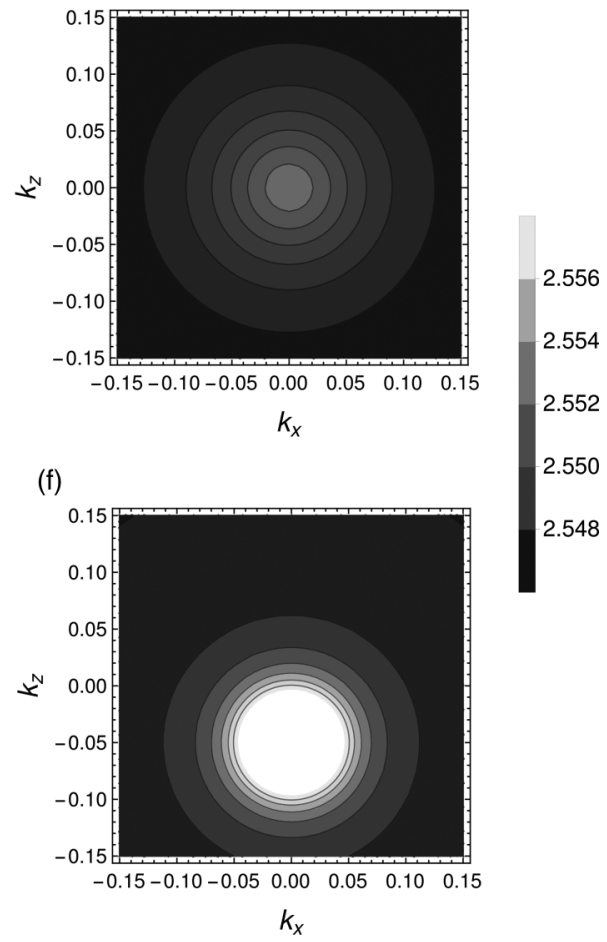

FIG. 3. Contour plots of the densities of states in the $k_{x}-k_{z}$ plane (with $k_{y}=0$ ) at a constant energy, $E$, slightly off zero. Plots from left to right correspond to increasing amplitude of the applied light for $\phi=0$ [(a)-(c)] and for $\phi=\pi / 2$ [(d)-(f)]. Plots in the top (bottom) row clearly show the dependence of the band gap (shift) on $\gamma$. In the top row, as $\gamma$ increases, the high DOS near the center of the $k_{x}-k_{z}$ plane (representing the degeneracy) decreases and gradually vanishes. In the bottom row, however, as $\gamma$ increases, the high DOS region shifts along $k_{z}$. Here we choose the following values for the different parameters: $\theta=\pi / 2, \omega=30, E=0.0025, \eta=0.00005, A_{y}=2$, and $A_{x}$ varying in integer steps from $0-2$ (left to right). As before, $k$ is dimensionless.

conductivity, $\Delta \sigma_{x y}$, at half filling using the relation [24]

$$
\Delta \sigma_{x y}=n \frac{e^{2}}{h} \frac{v_{z}}{2 \pi} .
$$

Here $n$ is the Chern number of the lower band and $v_{z}$ is the shift of the 3-BTP along the $k_{z}$ direction after applying light. Here we have restored factors of $\hbar, v_{f}$ and $e$ for ease of estimation. Thus, for our case we get

$$
\Delta \sigma_{x y}=2 \times \frac{e^{2}}{h} \times \frac{\gamma \sin 3 \phi}{2 \pi}=\frac{e^{2}}{h} \frac{\gamma \sin 3 \phi}{\pi}= \pm \frac{e^{2} \gamma}{\pi h} .
$$

As the monopole charge of our threefold fermion is twice that of a Weyl fermion, the resulting anomalous Hall signature also comes out to be double of its Weyl counterpart [41]. We will next discuss the values of different parameters required for experimental verification of our proposal and show that they lie well within current reach.

\section{F. Experimental considerations}

Now we estimate values for the change in Hall voltage and band gap likely to be measured in experiments. If our sample with transverse dimensions $l_{x} \times l_{y}$ and thickness $l_{z}=d$ is illuminated with a high frequency laser of power $P$ shining normally along $z$ direction, the penetration depth for light would be given by $\delta(\omega)=\frac{n(\omega) c \epsilon_{0}}{\Re \sigma_{x x}(\omega)}$, where $n$ is the refractive index of the material, $\sigma_{x x}$ is the longitudinal Hall conductivity, $c$ is the speed of light, and $\epsilon_{0}$ is the permittivity of free space. When a current $I_{x}$ is applied along $x$ direction, we can thus measure a Hall voltage $V_{y}$ along the perpendicular direction, given by $[24,33]$

$$
V_{y}=\frac{\sigma_{x y} \delta / d}{\sigma_{x x}^{2}+\left(\sigma_{x y} \delta / d\right)^{2}} \frac{I_{x}}{d} .
$$

As $\sigma_{x x} \gg \sigma_{x y}$, we can ignore the $\sigma_{x y}$ term in the denominator. The change in the Hall conductivity due to light will then read

$$
\Delta V_{y} \approx \frac{\Delta \sigma_{x y} \delta}{\sigma_{x x}^{2} d} \frac{I_{x}}{d}
$$

For circularly polarized light, $\sin \theta=1$ and $A_{x}=A_{y}=A$. Using $E \sim A \omega$, we thus get

$$
\gamma=\frac{e^{2} v_{f} A^{2}}{2 \hbar^{2} \omega}=\frac{e^{2} \hbar v_{f} E^{2}}{2(\hbar \omega)^{3}}=\frac{e^{2} \hbar v_{f}}{2(\hbar \omega)^{3}} \frac{2 P(1-R)}{l_{x} l_{y} n(\omega) c \epsilon_{0}},
$$

where $R$ is the reflectivity of the sample. This yields

$$
\Delta V_{y}=\left(\frac{e^{2}}{\pi h} \frac{e^{2} \hbar v_{f}}{2(\hbar \omega)^{3}} \frac{2 P(1-R)}{l_{x} l_{y} n(\omega) c \epsilon_{0}}\right)\left(\frac{n(\omega) c \epsilon_{0}}{\Re \sigma_{x x}(\omega)}\right) \frac{I_{x}}{\sigma_{x x}^{2} d^{2}} .
$$

We choose the typical sample parameters as $l_{x}=l_{y}=$ $100 \mu \mathrm{m}, d=100 \mathrm{~nm}, \sigma_{x x} \sim 10^{6} \Omega^{-1} \mathrm{~m}^{-1}, \Re \sigma_{x x}(\omega) \sim$ $10^{5} \Omega^{-1} \mathrm{~m}^{-1}$ and $v_{f} \sim 5 \times 10^{5} \mathrm{~m} / \mathrm{s}$ and consider shining a midinfrared laser pulse with $\omega=30 \mathrm{THz}$ and $P=1 \mathrm{~W}$ on 
(a)

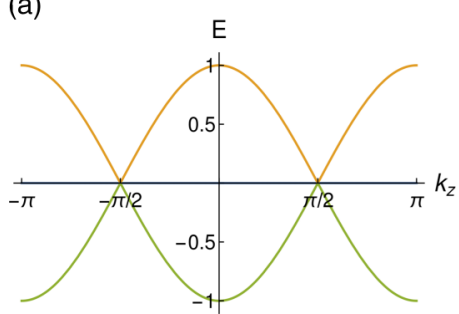

(b)

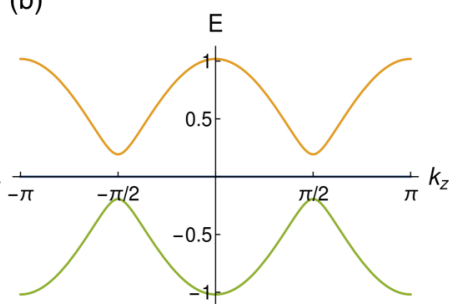

(c)

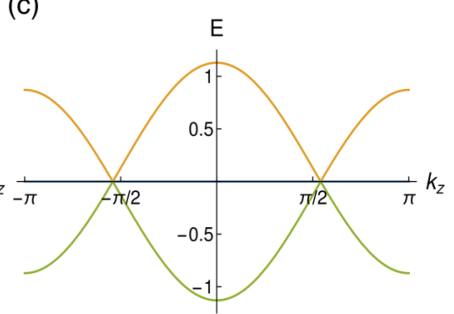

FIG. 4. Band-structure plots illustrating the effect of light on the lattice model. Eigenvalues of $H_{t b}$ (a) and $H_{F}$ (b), (c) are as function of $k_{z}\left(\right.$ at $\left.k_{x}=k_{y}=0\right)$. Plot (a) clearly shows the two threefold degenerate points at $\mathbf{k}=\left(0,0, \pm \frac{\pi}{2}\right)$ before applying light. After applying light, a band gap opens up for $\phi=\pi / 3$ (b), while the degeneracies shift along $k_{z}$ for $\phi=\pi / 2$ (c). This matches our low-energy model findings. Here we choose the following parameter values: $t=1, t_{z}=m=1, A_{x}=1, A_{y}=1$, and $\omega=3$. $E$ is expressed in units of $t$ while $k$ is expressed in units of $1 / a$ ( $a$ is the lattice constant which has been set to unity in our model).

our sample. With $R=0.8$ and $I_{x}=1$ A, we finally obtain $\Delta V_{y}=290 \mathrm{nV}$, which can be easily detected in experiment.

As the band gap is directly proportional to the intensity of applied light $I \sim P / l_{x} l_{y}$, using a laser with the same power $P=1 \mathrm{~W}$ but smaller spot size would lead to larger effect on the band structure. With $l_{x}=l_{y}=1 \mu \mathrm{m}$, we get $\Delta_{g}=$ $2 \hbar v_{f} \gamma \approx 25 \mathrm{meV}$, which can again be readily measured in current ARPES experiments.

Heating in Floquet systems is a major concern for experimental realization. However, there are some known ways of evading the problem [45]. Opening a gap above the conduction and below the valence bands [28] is a possible way of preventing heating. Additionally, Floquet phases can be supported in prethermal regimes, where heating takes a parametrically long time [46-48]. Finally, driven systems can be actively cooled by coupling to a bath. Under suitable conditions, Floquet phases can be stabilized in such systems [49-52].

\section{LATTICE MODEL}

Next we consider a lattice model having two triply degenerate points of opposite monopole charge in the first Brillouin zone to investigate the nature of the Fermi arcs and the effect of light on them.

We start with a simplified tight binding model for triplecomponent fermions without spin-orbit coupling (or $\phi=\pi / 2$ ) [53]. The Hamiltonian reads

$$
\begin{aligned}
H(\mathbf{k})= & t \sin k_{x} S_{x}+t \sin k_{y} S_{y} \\
& +\left[t_{z} \cos k_{z}+m\left(\cos k_{x}+\cos k_{y}-2\right)\right] S_{z},
\end{aligned}
$$

where $S=\left(S_{x}, S_{y}, S_{z}\right)$ is a spin-1 representation of $\mathrm{SU}(2)$ :

$$
\boldsymbol{S}=i\left[\begin{array}{ccc}
0 & \hat{e_{x}} & -\hat{e_{y}} \\
-\hat{e_{x}} & 0 & \hat{e}_{z} \\
\hat{e_{y}} & -\hat{e}_{z} & 0
\end{array}\right]
$$

In the Hamiltonian, the $\cos k_{z}$ term vanishes at $k_{z}= \pm \pi / 2$. We work with the case where $t_{z}=m$ such that the mass term can vanish at $\left(k_{x}, k_{y}\right)=(0,0)$ where the coefficients of $S_{x}$ and $S_{y}$ also go to zero. With the above constraint, the system possess two threefold degeneracies at $\mathbf{k}=\left(0,0, \pm \frac{\pi}{2}\right)$ in the band structure. We generalize this lattice model for all values of $\phi$ and write the generalized Hamiltonian, $H_{t b}$, as

$$
H_{t b}(\mathbf{k})=\left[\begin{array}{ccc}
0 & e^{i \phi} t \sin k_{x} & e^{-i \phi} t \sin k_{y} \\
e^{-i \phi} t \sin k_{x} & 0 & e^{i \phi} m\left(\cos k_{z}+\cos k_{x}+\cos k_{y}-2\right) \\
e^{i \phi} t \sin k_{y} & e^{-i \phi} m\left(\cos k_{z}+\cos k_{x}+\cos k_{y}-2\right) & 0
\end{array}\right] .
$$

The eigenvalues of $H_{t b}(\mathbf{k})$ are plotted in Fig. 4(a), clearly showing the two 3-BTPs at $\mathbf{k}=\left(0,0, \pm \frac{\pi}{2}\right)$. We note that in the limit of $\mathbf{k} \rightarrow\left(0,0,-\frac{\pi}{2}\right)$, we recover exactly our low energy Hamiltonian, $H_{3 f}$, as given by Eq. (1). The band structure close to $k_{z}= \pm \frac{\pi}{2}$ also faithfully retains all the properties of the low-energy Hamiltonian discussed above.

\section{A. Floquet analysis for the tight-binding model}

Next, we examine the properties of the tight-binding model upon illumination. When we shine light on our lattice system, we can again use Floquet theory to study the resulting effective band structures. From our analysis of the low-energy model, we know that linearly polarized light cannot change the band structure while elliptically polarized light affects it the most. We thus choose $\theta=\pi / 2$, giving the vector potential of light as $\boldsymbol{A}=\left(A_{x} \cos \omega t,-A_{y} \sin \omega t, 0\right)$. After some calculations, we find the lowest order light induced term to be

$$
\frac{\left[V_{+1}, V_{-1}\right]}{\omega}=i \zeta\left[\begin{array}{ccc}
0 & -e^{-2 i \phi} m t \sin k_{x} \cos k_{y} & e^{2 i \phi} m t \sin k_{y} \cos k_{x} \\
e^{2 i \phi} m t \sin k_{x} \cos k_{y} & 0 & -e^{-2 i \phi} t^{2} \cos k_{x} \cos k_{y} \\
-e^{-2 i \phi} m t \sin k_{y} \cos k_{x} & e^{2 i \phi} t^{2} \cos k_{x} \cos k_{y} & 0
\end{array}\right],
$$


(a)

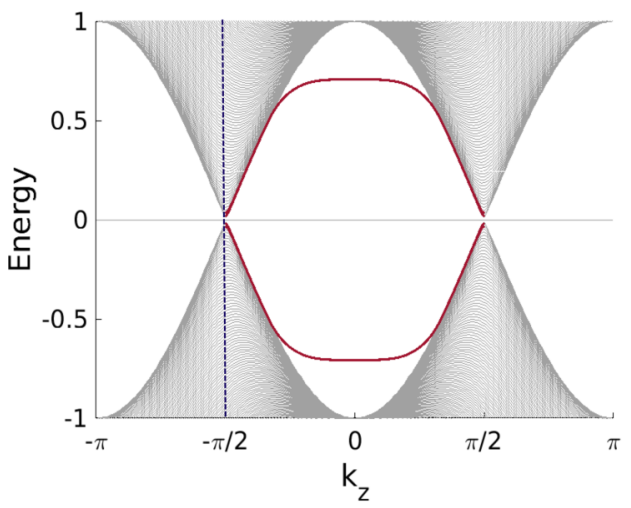

(c)

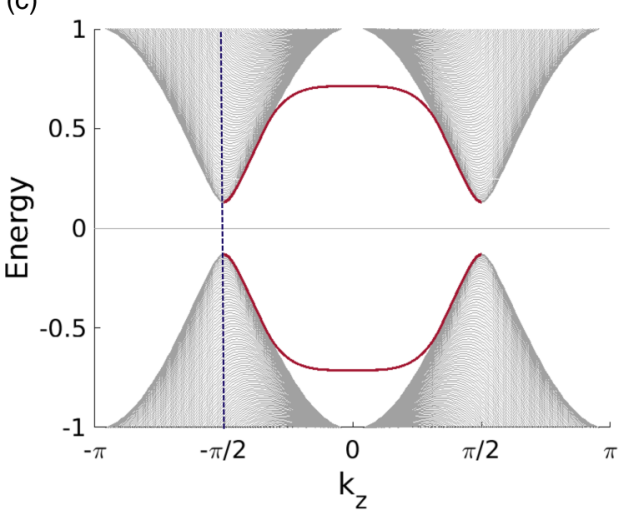

(b)

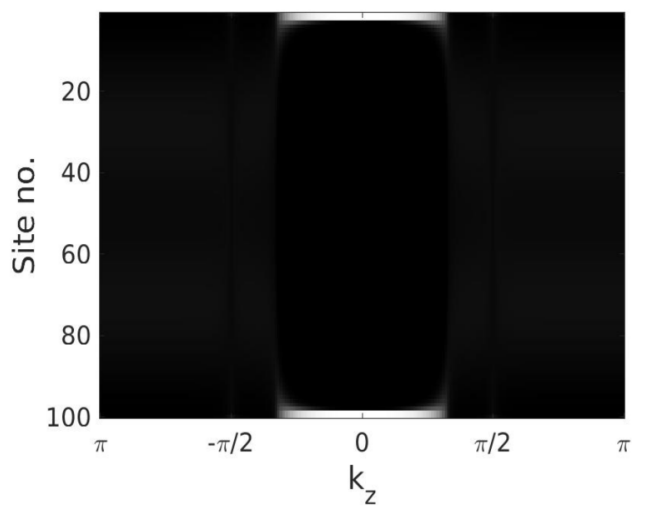

(d)

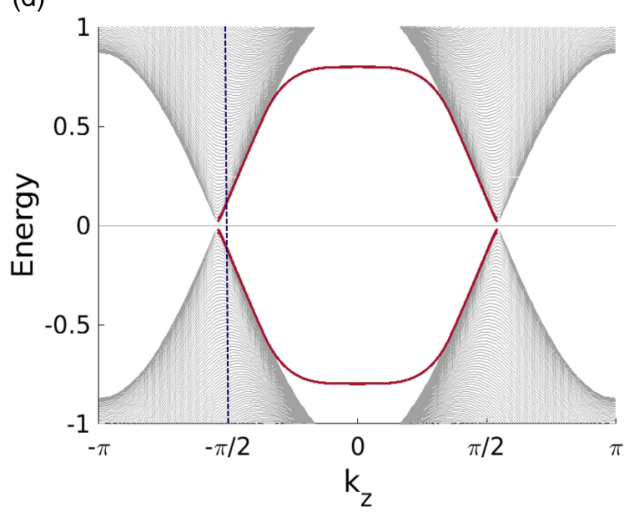

FIG. 5. Numerically calculated band structure for the lattice model in a slab geometry (a) without light and (c)-(d) with light. We consider a slab with 200 sites along the $x$ direction having periodic boundary conditions along the $y$ and $z$ directions. All eigenvalues have been plotted along $k_{z}\left(\right.$ at $\left.k_{y}=0\right)$. Surface states arising due to the non-zero Chern number of the bands have been highlighted in red. The sum of distributions of their corresponding wave functions is shown in (b). They are highly surface localized near $k_{z}=0$ but start penetrating into the bulk as the surface states mix with the bulk bands. Without application of light [panel (a)], the surface states connect the two threefold degeneracies. Under illumination with light, the surface states connect the shifted degeneracies for $\phi=\pi / 2$ [panel (c)], while they do not traverse the bulk gap for $\phi=0$ [panel (d)]. Here we use the following parameter values: $t=1, t_{z}=m=1, A_{x}=1, A_{y}=1$, and $\omega=3$. $E$ is expressed in units of $t$ while $k$ is expressed in units of $1 / a$.

with $\zeta$ defined as

$$
\zeta=\frac{2 J_{1}\left(A_{x}\right) J_{1}\left(A_{y}\right)}{\omega},
$$

where $J_{1}$ is the Bessel function of the first kind. The photondressed effective Hamiltonian reads $H_{F}=H_{t b}+\frac{\left[V_{+1}, V_{-1}\right]}{\omega}$. Plots of the eigenvalues of $H_{F}$ are presented in Figs. 4(b) and 4(c). From the band structure, we observe that a band gap opens up and/or the two 3-BTPs shift along the $k_{z}$ axis depending on the value of $\phi$, similar to the case of the low-energy model. The pattern exactly matches the one shown in Fig. 2, in agreement with our low-energy effective model analysis. For $\phi \in\left(0, \frac{\pi}{3}\right)$, the two 3-BTPs shift inward toward $k_{z}=0$, while for $\phi \in\left(\frac{\pi}{3}, \frac{2 \pi}{3}\right)$, they shift outward, and the pattern continues in a periodic fashion.

\section{B. Surface states and Fermi arcs}

As our system has a nontrivial topology, we expect to observe topologically protected surface states representing the bulk-boundary correspondence. To investigate these surface states and the effect of light on them, we consider our gener- alized tight-binding model under open boundary conditions. We make our lattice finite along the $x$ direction, while keeping it periodic along the $y$ and $z$ directions. The numerical results for the band structure are shown in Fig. 5(a). Apart from the bulk bands, we find that surface states connecting the two 3-BTPs appear in this slab geometry. As shown in Fig. 5(b), their corresponding wave functions are highly localized near the surface for $k_{z}$ close to zero but start penetrating into the bulk as the surface states mix with the bulk bands. Both the upper and the lower bands contribute two such states, in agreement with the bulk Chern number for the two bands being \pm 2 .

After applying light, we observe that the surface states remain intact [see Figs. 5(c) and 5(d)], indicating that light does not change the Chern number of the bands. The structure of the surface states remains preserved for $\phi=\pi / 6(\bmod$ $\pi / 3$ ), however, they now connect the shifted 3-BTPs. For other values of $\phi$ where a gap opens up, the surface states do not traverse the bulk gap. The wave function for these surface states, however, does not show any significant change with respect to surface localization and decay upon illumination with light. 

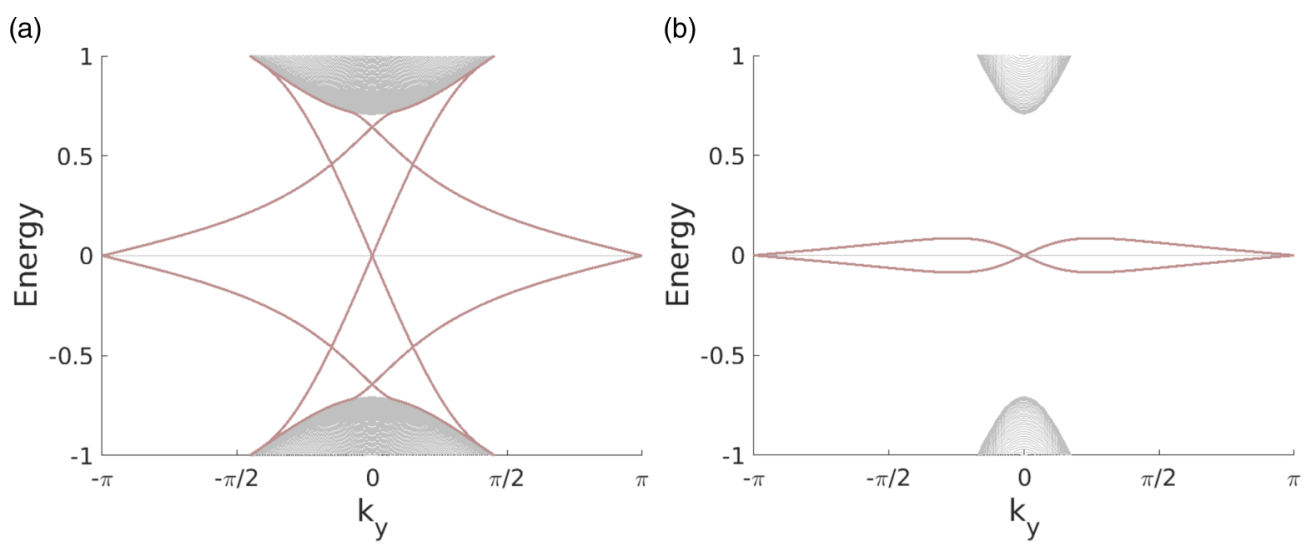

FIG. 6. Illustration of the anomalous Hall insulator planes that stack to give rise to multifold fermion semimetal. (a) A plane at $k_{z}=\pi / 4$ representing all planes in $k_{z} \in(-\pi /, \pi / 2)$. (b) A plane at $k_{z}=3 \pi / 4$ representing all planes in $k_{z} \in(-\pi,-\pi / 2) \cup(\pi / 2, \pi)$. The band structure has been numerically calculated for the lattice model in a slab geometry similar to Fig. 5. Surface states arising due to the slab geometry are highlighted in red. In panel (a), we can see chiral surface states traversing the gap, whereas in panel (b) no such surface states are present. As before, we choose the following parameter values: $t=1, t_{z}=m=1, A_{x}=1, A_{y}=1$, and $\omega=3$. Again, $E$ is expressed in units of $t$ while $k$ is expressed in units of $1 / a$.

\section{Anomalous Hall conductivity}

We now move on to calculate the change in the anomalous Hall signature for our lattice model for $\phi=\pi / 2$ and compare it to our low energy effective model results. Analogous to a Weyl semimetal $[6,54]$, our threefold semimetal can be thought of as being constructed by stacking planes of anomalous Hall insulators with Chern numbers \pm 2 along $k_{z}$ [53]. We show two such anomalous Hall insulator planes in Fig. 6. For $k_{z} \in(-\pi / 2, \pi / 2)$, each plane possesses chiral surface states traversing the gap which show a quantized Hall conductance. However, for planes beyond $\pi / 2$, no such surface states are present. Thus, at half filling the semimetal would show a large anomalous Hall conductivity coming from contributions from each of the planes between the two 3-BTPs implying $\sigma_{x y}=2 \times \frac{e^{2}}{h} \times \frac{K_{0}}{2 \pi}$ where $K_{0}$ is the separation between the two 3-BTPs. This gives $\sigma_{x y}=\frac{e^{2}}{h}$.

When light is applied, $K_{0}$ changes, leading to a change in the anomalous Hall signature. To get an analytical form for the 3-BTP shift, we consider the mass term in $H_{t b}$ at $k_{x}=k_{y}=0$, which reads $\cos k_{z}+\zeta$. For small $\zeta$, the mass term vanishes for $k_{z}= \pm\left(\pi / 2+\delta k_{z}\right)$. From the condition, $-\zeta=\cos \left(\pi / 2+\delta k_{z}\right)$, where $\cos \left(\pi / 2+\delta k_{z}\right) \approx-\delta k_{z}^{2} / 2$, we thus obtain $\delta k_{z}=\zeta$. This tells us that the Hall signature changes by

$$
\Delta \sigma_{x y}=2 \times \frac{e^{2}}{h} \times \frac{2 \zeta}{2 \pi}=\frac{2 e^{2} \zeta}{\pi h} .
$$

The contribution from each node $\sim \frac{e^{2} \zeta}{\pi h}$ is similar to the low-energy model results. Such a change in the anomalous Hall conductivity of the threefold fermion material upon illumination could be measured directly to test our predictions.

\section{SUMMARY}

We have studied the effect of light on triplefold fermions, employing an effective low-energy model and a simplified tight-binding model using the Floquet-Magnus expansion. We have shown that elliptically polarized light can cause a band gap to open and/or shift the threefold degeneracy depending on the intrinsic symmetries of the system. We show how the shift in the degeneracy changes the anomalous Hall conductivity causing an experimentally detectable change in the Hall voltage. We have also discussed the experimental feasibility of our proposals. We hope that our work further motivates exploration of light-matter interaction in multifold fermions.

\section{ACKNOWLEDGMENTS}

We thank Barry Bradlyn (University of Illinois at UrbanaChampaign) and Snehasish Nandy (University of Virginia) for useful discussions. R.J. thanks the Kishore Vaigyanik Protsahan Yojana (KVPY) for a fellowship. A.N. acknowledges support from startup Grant No. SG/MHRD-19-0001 of the Indian Institute of Science.

\section{APPENDIX A: VALIDITY OF FLOQUET APPROXIMATION}

In the Floquet expansion for the low-energy model, the various terms in increasing powers of $(1 / \omega)$ have the form $H_{0}, V \frac{V}{\hbar \omega}, V\left(\frac{V}{\hbar \omega}\right)^{2}$, and so on. Keeping terms only up to first order in $V / \hbar \omega$ is reasonable if $\omega \gg \frac{V}{\hbar} \approx \frac{e v_{f} A}{\hbar}$. Using $E \sim A \omega$, where $E$ is the root-mean-squared value of the electric field of light, we get the condition $\omega \gg \frac{e v_{f} E}{\hbar \omega}$ or $\omega \gg \sqrt{\frac{e v_{f} E}{\hbar}}$. Now $E \sim \sqrt{\frac{2 I(1-R)}{n c \epsilon_{0}}}$, where $I$ is the intensity of applied light. With the different parameters as given in Sec. IIF, we get the condition on the possible applied frequencies as $\omega \gg 1 \mathrm{THz}$.

\section{APPENDIX B: ANALYTICAL FORM OF THE ENERGY BANDS}

The eigenvalues for $H_{\text {floq }}$ read

$$
\begin{aligned}
& E_{+}=\frac{2 \hbar v_{f}}{\sqrt{3}} \sqrt{\lambda_{1}} \cos \left(\frac{\psi-\pi}{3}\right) \\
& E_{-}=-\frac{2 \hbar v_{f}}{\sqrt{3}} \sqrt{\lambda_{1}} \cos \left(\frac{\psi}{3}\right)
\end{aligned}
$$




$$
\begin{aligned}
E_{0} & =\frac{2 \hbar v_{f}}{\sqrt{3}} \sqrt{\lambda_{1}} \cos \left(\frac{\psi+\pi}{3}\right), \\
\text { with } \psi & = \begin{cases}\tan ^{-1}\left(\frac{\sqrt{4 \lambda_{1}^{3}-\lambda_{2}^{2}}}{\lambda_{2}}\right) & \text { if } \lambda_{2}>0 \\
\pi / 2 & \text { if } \lambda_{2}=0 \\
\tan ^{-1}\left(\frac{\sqrt{4 \lambda_{1}^{3}-\lambda_{2}^{2}}}{\lambda_{2}}\right)+\pi & \text { if } \lambda_{2}<0,\end{cases}
\end{aligned}
$$

$$
\text { where } \begin{aligned}
\lambda_{1} & =|k|^{2}+\gamma^{2}-2 k_{z} \gamma \sin 3 \phi \quad \text { and } \\
\lambda_{2} & =-54 k_{x} k_{y} k_{z} \cos 3 \phi
\end{aligned}
$$

At special values of $\phi=\frac{\pi}{6}(\bmod \pi / 3)$, we recover the eigenvalues $E_{ \pm}=0, \pm \hbar v_{f} \sqrt{k_{x}^{2}+k_{y}^{2}+\left(k_{z}-\gamma / \hbar\right)^{2}}$ as discussed in the text.
[1] K. v. Klitzing, G. Dorda, and M. Pepper, Phys. Rev. Lett. 45, 494 (1980).

[2] D. J. Thouless, M. Kohmoto, M. P. Nightingale, and M. den Nijs, Phys. Rev. Lett. 49, 405 (1982).

[3] M. Z. Hasan and C. L. Kane, Rev. Mod. Phys. 82, 3045 (2010).

[4] X.-L. Qi and S.-C. Zhang, Rev. Mod. Phys. 83, 1057 (2011).

[5] A. A. Burkov, Nat. Mater. 15, 1145 (2016).

[6] N. P. Armitage, E. J. Mele, and A. Vishwanath, Rev. Mod. Phys. 90, 015001 (2018).

[7] B. Bradlyn, J. Cano, Z. Wang, M. G. Vergniory, C. Felser, R. J. Cava, and B. A. Bernevig, Science 353, aaf5037 (2016).

[8] G. Chang, S.-Y. Xu, B. J. Wieder, D. S. Sanchez, S.-M. Huang, I. Belopolski, T.-R. Chang, S. Zhang, A. Bansil, H. Lin, and M. Z. Hasan, Phys. Rev. Lett. 119, 206401 (2017).

[9] D. Takane, Z. Wang, S. Souma, K. Nakayama, T. Nakamura, H. Oinuma, Y. Nakata, H. Iwasawa, C. Cacho, T. Kim, K. Horiba, H. Kumigashira, T. Takahashi, Y. Ando, and T. Sato, Phys. Rev. Lett. 122, 076402 (2019).

[10] Z. Rao, H. Li, T. Zhang, S. Tian, C. Li, B. Fu, C. Tang, L. Wang, Z. Li, W. Fan, J. Li, Y. Huang, Z. Liu, Y. Long, C. Fang, H. Weng, Y. Shi, H. Lei, Y. Sun, T. Qian, and H. Ding, Nature 567, 496 (2019).

[11] N. B. M. Schröter, D. Pei, M. G. Vergniory, Y. Sun, K. Manna, F. de Juan, J. A. Krieger, V. Süss, M. Schmidt, P. Dudin, B. Bradlyn, T. K. Kim, T. Schmitt, C. Cacho, C. Felser, V. N. Strocov, and Y. Chen, Nat. Phys. 15, 759 (2019).

[12] N. Kumar, M. Yao, J. Nayak, M. G. Vergniory, J. Bannies, Z. Wang, N. B. M. Schröter, V. N. Strocov, L. Müchler, W. Shi, E. D. L. Rienks, J. L. Mañes, C. Shekhar, S. S. P. Parkin, J. Fink, G. H. Fecher, Y. Sun, B. A. Bernevig, and C. Felser, Adv. Mater. 32, 1906046 (2020).

[13] M.-A. Sánchez-Martínez, F. de Juan, and A. G. Grushin, Phys. Rev. B 99, 155145 (2019).

[14] F. Flicker, F. de Juan, B. Bradlyn, T. Morimoto, M. G. Vergniory, and A. G. Grushin, Phys. Rev. B 98, 155145 (2018).

[15] D. Rees, K. Manna, B. Lu, T. Morimoto, H. Borrmann, C. Felser, J. E. Moore, D. H. Torchinsky, and J. Orenstein, Sci. Adv. 6, eaba0509 (2020).

[16] Z. Ni, K. Wang, Y. Zhang, O. Pozo, B. Xu, X. Han, K. Manna, J. Paglione, C. Felser, A. G. Grushin, F. de Juan, E. J. Mele, and L. Wu, arXiv:2006.09612.

[17] Z. Ni, B. Xu, M. A. Sanchez-Martinez, Y. Zhang, K. Manna, C. Bernhard, J. W. F. Venderbos, F. de Juan, C. Felser, A. G. Grushin, and L. Wu, [npj Quantum Mater. (to be published)], arXiv:2005.13473.

[18] J. Cayssol, B. Dóra, F. Simon, and R. Moessner, Phys. Status Solidi RRL 7, 101 (2013).
[19] U. D. Giovannini and H. Hübener, J. Phys.: Mater. 3, 012001 (2019).

[20] T. Oka and S. Kitamura, Annu. Rev. Condens. Matter Phys. 10, 387 (2019).

[21] T. Oka and H. Aoki, Phys. Rev. B 79, 081406(R) (2009).

[22] N. H. Lindner, G. Refael, and V. Galitski, Nat. Phys. 7, 490 (2011).

[23] T. Kitagawa, T. Oka, A. Brataas, L. Fu, and E. Demler, Phys. Rev. B 84, 235108 (2011).

[24] C.-K. Chan, P. A. Lee, K. S. Burch, J. H. Han, and Y. Ran, Phys. Rev. Lett. 116, 026805 (2016).

[25] M. Ezawa, Phys. Rev. Lett. 110, 026603 (2013).

[26] A. Narayan, Phys. Rev. B 91, 205445 (2015).

[27] Y. H. Wang, H. Steinberg, P. Jarillo-Herrero, and N. Gedik, Science 342, 453 (2013).

[28] J. W. McIver, B. Schulte, F.-U. Stein, T. Matsuyama, G. Jotzu, G. Meier, and A. Cavalleri, Nat. Phys. 16, 38 (2020).

[29] A. López and R. A. Molina, J. Phys.: Condens. Matter 32, 205701 (2020).

[30] H. Hübener, M. A. Sentef, U. De Giovannini, A. F. Kemper, and A. Rubio, Nat. Commun. 8, 13940 (2017).

[31] R. Wang, B. Wang, R. Shen, L. Sheng, and D. Y. Xing, Europhys. Lett. 105, 17004 (2014).

[32] A. Narayan, Phys. Rev. B 94, 041409(R) (2016).

[33] Z. Yan and Z. Wang, Phys. Rev. Lett. 117, 087402 (2016).

[34] C.-K. Chan, Y.-T. Oh, J. H. Han, and P. A. Lee, Phys. Rev. B 94, 121106(R) (2016).

[35] A. G. Grushin, A. Gómez-León, and T. Neupert, Phys. Rev. Lett. 112, 156801 (2014).

[36] K. Takasan, A. Daido, N. Kawakami, and Y. Yanase, Phys. Rev. B 95, 134508 (2017).

[37] G. E. Topp, G. Jotzu, J. W. McIver, L. Xian, A. Rubio, and M. A. Sentef, Phys. Rev. Research 1, 023031 (2019).

[38] L. He, Z. Addison, J. Jin, E. J. Mele, S. G. Johnson, and B. Zhen, Nat. Commun. 10, 4194 (2019).

[39] J. Guglielmon, S. Huang, K. P. Chen, and M. C. Rechtsman, Phys. Rev. A 97, 031801(R) (2018).

[40] A. Banerjee and A. Narayan, Phys. Rev. B 102, 205423 (2020).

[41] S. K. Firoz Islam and A. A. Zyuzin, Phys. Rev. B 100, 165302 (2019).

[42] J.-T. Wu, X.-H. Shi, and Y.-J. Wei, Acta Mech. Sin. 28, 1539 (2012).

[43] C.-K. Chan, N. H. Lindner, G. Refael, and P. A. Lee, Phys. Rev. B 95, 041104(R) (2017).

[44] H. Bruus and K. Flensberg, Many-Body Quantum Theory in Condensed Matter Physics: An Introduction, Oxford Graduate Texts (Oxford University Press, Oxford, 2004). 
[45] F. Harper, R. Roy, M. S. Rudner, and S. Sondhi, Annu. Rev. Condens. Matter Phys. 11, 345 (2020).

[46] T. Kuwahara, T. Mori, and K. Saito, Ann. Phys. 367, 96 (2016).

[47] S. A. Weidinger and M. Knap, Sci. Rep. 7, 45382 (2017).

[48] D. Abanin, W. De Roeck, W. W. Ho, and F. Huveneers, Commun. Math. Phys. 354, 809 (2017).

[49] T. Iadecola, T. Neupert, and C. Chamon, Phys. Rev. B 91, 235133 (2015).

[50] H. Dehghani, T. Oka, and A. Mitra, Phys. Rev. B 91, 155422 (2015).
[51] K. I. Seetharam, C.-E. Bardyn, N. H. Lindner, M. S. Rudner, and G. Refael, Phys. Rev. X 5, 041050 (2015).

[52] K. Seetharam, P. Titum, M. Kolodrubetz, and G. Refael, Phys. Rev. B 97, 014311 (2018).

[53] S. Nandy, S. Manna, D. Călugăru, and B. Roy, Phys. Rev. B 100, 235201 (2019).

[54] B. A. Bernevig and T. L. Hughes, Topological Insulators and Topological Superconductors (Princeton University Press, Princeton, 2013). 